\title{
Pole Length Influences Performance During On-Snow Skating in Female Cross-Country Skiers
}

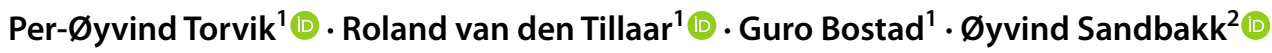

Received: 17 March 2020 / Accepted: 24 July 2021 / Published online: 27 October 2021

(c) The Author(s) 2021

\begin{abstract}
Purpose The purpose of this study was to examine the effect of pole length on performance and technique selection during a simulated skating cross-country (XC) skiing competition on snow in female XC skiers.

Methods Nine female XC skiers and biathletes $\left(\mathrm{VO}_{2 \max } 63.6 \pm 6.2 \mathrm{~mL} / \mathrm{min} / \mathrm{kg}\right.$, age $22.9 \pm 3.5$ years, body height $1.69 \pm 0.1 \mathrm{~m}$ and body mass $60.8 \pm 4.6 \mathrm{~kg}$ ) completed two 5 - $\mathrm{km}$ skating time-trail with maximal effort. The athletes had a minimum $4.5 \mathrm{~h}$ of rest between the two races, which were performed in a random order: one with self-selected poles $(89.0 \% \pm 0.6 \%$ of body height) and one with $7.5 \mathrm{~cm}$ increased pole length $(94.0 \% \pm 0.5 \%$ of body height). Speed in set terrain sections was determined and the selection of sub-technique was self-reported immediately after each race based on a detailed review of the entire track. Results Skiers performed on average $7.1 \pm 7.1 \mathrm{~s}(P=0.029)$ faster with the long poles, with this difference occurring during the first $200 \mathrm{~m}$ and in the uphill parts of the track, in which $\sim 5 \%$ more G3 and 5\% fewer G2 sub-techniques were chosen (both $P<0.05)$. The rating of perceived exertion was $1 \pm 0.9$ point lower $(P=0.04)$ and skiing technique was perceived to be $\sim 1.2 \pm 1.5$ points better with long poles $(P=0.038)$, while the physiological responses (i.e., peak and average heart rate, and blood lactate concentration) did not differ between trials.

Conclusion In conclusion, poles $7.5 \mathrm{~cm}$ longer than self-selected ones improved performance in skating, by enhancing speed in the initial phase (first $200 \mathrm{~m}$ ) and in the uphill section of the track. In addition, the longer poles induced more use of the G3 skating sub-technique.
\end{abstract}

Keywords XC skiing $\cdot$ Skiing performance $\cdot$ Skiing equipment $\cdot$ Sub-technique selection

\section{Introduction}

In cross-country (XC) skiing, skiers propel themselves forward by combining ski push-offs and poling. Accordingly, the characteristics of the skis and poles are crucial for the effectiveness of most skiing technique in both classical and skating styles. Previously, the use of longer poles has been shown to increase skiing efficiency and performance in double poling $[5,13,14,17]$ and in the G3 skating technique on roller skis [29]. Moreover, two previous studies showed positive effects of increased pole length on snow in the classical

Per-Øyvind Torvik

per-o-torvik@nord.no

1 Department of Sports Sciences and Physical Education, Nord University, Levanger, Norway

2 Centre for Elite Sports Research, Department of Neuromedicine and Movement Science, Norwegian University of Science and Technology, Trondheim, Norway style $[8,30]$. Although there are indications from the abovementioned study on G3 roller ski skating [29] that increased pole length could be beneficial in skating, at least in some of the sub-techniques, this has not yet been examined while ski skating on snow.

The use of longer poles in the classical style together with better equipment and preparation of the track [21] as well as a stronger and more endurance trained upper body [18] have contributed to make double poling one of the most favored classical sub-techniques used in races. Today double poling is widely employed even in uphill sections by both male and female cross-country skiers $[5,9,17$, 24]. In classical races, the benefits of longer poles led to restrictions in pole length and the inclusion of diagonal zones in competitions [6]. However, the possible benefits of longer poles in the skating technique have not been systematically evaluated and the poles in skating are only limited to the skier's body height [6]. 
A potential effect on performance of increased pole length may be associated with changes in the fractional use of the five different skating sub-techniques (G1-5). The G2, G3 and G4 techniques are the most favored subtechniques in skating $[12,16]$. Among these, the two most investigated sub-techniques are $\mathrm{G} 2[2,4,11,22,26]$, which is mainly used on steep uphill stretches and when accelerating, and G3, a technique normally used on flat and slightly uphill terrain. Interestingly, Myklebust et al. [15] reported that the G3 technique was limited by the same factors as double poling, especially concerning the way potential energy is gained between pole plants, the propulsive force in the poling action, and the conformity in upper-body muscle work. This highlights a particular potential to enhance G3 speed and thereby use this sub-technique over a wider range of terrain using longer poles. The mechanisms behind enhanced performance and reduced $\mathrm{O}_{2}$ cost of longer poles in double poling has recently been examined by several researchers [5, 13, 17], showing that double poling with longer poles in low to moderate uphill terrain resulted in reduced vertical displacement of the center of mass (CoM), and longer poling time. To what extent these findings also apply to the skating technique has not yet been examined.

The question of finding the optimal pole length in skating has been a "hot topic" since skiers in 1985 started to use the skating technique systematically during World Cup events [1], and until now 7.5-10 cm longer poles ( $90 \%$ of body height) than those used in classical style have been regarded as beneficial. However, this has been sparsely evaluated scientifically, and to our knowledge has not changed significantly since 1985 . Longer poles have certainly not been systematically evaluated in female XC skiers despite the fact that longer pole lengths are now considered more effective in uphill roller ski skating on a treadmill [29] in double poling [5, 17] but not in the other classical sub-techniques [30], which may indicate that a positive effect could also apply to skating. Trøen et al. [30], however, included seven females in his studies of classic ski technique on snow and reported an even greater effect for females than males in double poling with longer poles. However, the potential benefit of using longer poles in skating should be further explored on snow, including how pole length affects the skier's choice of different subtechniques across hilly terrain.

Therefore, this study investigated the effect of increased pole length on performance and selection of sub-technique during on-snow ski skating in female skiers. It was hypothesized that longer poles would lead to improved performance through greater use of the G3 skating technique.

\section{Materials and Methods}

\section{Participants}

Nine competitive female junior and senior skiers (maximal oxygen uptake in running: $63.6 \pm 6.2 \mathrm{~mL} / \mathrm{min} / \mathrm{kg}$, FIS points: $100.2 \pm 33.2$, maximal heart rate: $197 \pm 7$ beats/ min, age: $22.9 \pm 3.5$ years, height: $1.69 \pm 0.1 \mathrm{~m}$ and body mass: $60.8 \pm 4.6 \mathrm{~kg}$ ), participated in this study. The participants had competed at national and international level for $6.0 \pm 2.0$ years. They were not familiar with using longer poles than their regular ones at the time of the study. The participants provided written informed consent to participate in the study, which was recommended by the Norwegian Center for Research Data and performed according to the Declaration of Helsinki. All participants were 18 years or older at the start of the study.

\section{Design}

To compare the effects of self-selected and longer (selfselected $+7.5 \mathrm{~cm}$ ) skating poles upon skiing performance, selection of sub-technique, as well as physiological and perceptual responses during a simulated competition, two 5-km time trial races on an internationally approved track were carried out on the same day using a randomized cross-over design, acting like their own controls. Five skiers started with the self-selected poles and four started with the $+7.5 \mathrm{~cm}$ poles. All skiers had a minimum of $4.5 \mathrm{~h}$ rest between each time trial. On separate days, 5-7 days before the time trials, all skiers were tested for maximal oxygen uptake $\left(\mathrm{VO}_{2 \max }\right)$ in the laboratory and maximal heart rate $\left(\mathrm{HR}_{\max }\right)$ in uphill running outdoors. $\mathrm{VO}_{2 \max }$ was tested to describe the aerobic endurance level of the athletes in a standard incremental uphill running test at $10.0 \%$ inclination on a motor-driven treadmill, with increasing speed every minute until exhaustion, with a protocol and procedures published previously [28]. Similarly, maximal heart rate was tested in an outdoor uphill running test, also with a protocol and procedures published previously [10].

\section{Procedure}

The participants prepared for all tests as they would for a regular competition [31], except that a standard warm-up was performed. In the $24 \mathrm{~h}$ preceding all tests, the participants were instructed to eat their typical diet when preparing for a competition, and had to avoid strenuous exercise, caffeine, and alcohol. Each participant arrived in the laboratory or at the ski venue one hour before each test for a short interview, 
to ensure that they were well nourished, hydrated, motivated and healthy. A standard warm-up, consisting of 15-min running at $\sim 60 \%-70 \%$ of maximum HR was performed before all tests. The subjects had at least five hours between each time trial and were instructed to have total rest in the final two hours before warm-up for test 2.

The time trial tests were performed as simulated $5-\mathrm{km}$ competitions in the skating technique, with participants with the lowest FIS points starting first, and a 1-min start interval, to avoid any interference between athletes. The participants started randomly with self-selected or longer poles. The selfselected pole length was $89.0 \% \pm 1.0 \%$ of the participant's body height, while the longer pole length was $94.0 \% \pm 1.0 \%$ of body height. Other ski equipment was individualized to the specific skiers' racing preferences, including racing suits, boot style, ski length and ski base material. All skiers were instructed to use their own skis for the prevailing conditions; these were only used for the two time trials, while other skis were used for warming up and cooling down. All skis were stone ground and prepared with a $1 \mathrm{~mm}$ hand structured linear roller (Swix, Norway) on the back of each ski. All skis were prepared with an LF 7 glide wax (Swix, Norway), and an LDF liquid glide topping (Vauhti, Finland) was used before each time trial. The track was prepared with a Pisten Bully 600 snowmobile, and the snow was hard-packed with similar conditions during the entire experiment. The weather conditions were measured at the start of each race with a digital weather station (Metnet, Norway), and weather and snow conditions were stable during the test day. The air temperature during the test day ranged between $-2.0^{\circ}$ and $-0.9^{\circ}$, and the snow temperature from $-4.0^{\circ}$ to $-4.7^{\circ}$ (T95 Swix Snow Thermometer, Norway). The wind was $0-2 \mathrm{~m} / \mathrm{s}$ from the north-east, the barometric pressure was from 1055 to $1079 \mathrm{mmHg}$ and there was thin high cloud the whole day. Possible changes in the coefficient of snow ski friction were not measured between the two time trials, but the time spent in the longest straight downhill segment was used as an indirect measure of friction, which revealed no differences.

Heart rate was measured continuously and the average heart rate for the entire $5 \mathrm{~km}$ was determined. At the end of each time trial, blood lactate concentration and rate of perceived exertion (RPE) were assessed. Finally, the skiers were asked to evaluate their experience of skiing with the longer poles, when compared to the self-chosen poles, on a custom-made scale from 1 to 10 , where 10 was defined as a much better feeling, 5 no difference and 1 as a much worse feeling. The evaluation applied to each of the five sections of the track.

\section{Instruments and Measurements}

In the outdoor time trial test, the blood lactate concentration of $5-\mu \mathrm{L}$ samples was taken from the fingertip and analyzed using a Lactate Pro LT-1710t kit (Arkray Inc.,
Kyoto, Japan). The subjects' RPE was recorded using the Borg (6-20) scale [17]. Course and elevation profiles were determined with a Catapult Optimeye S5 (Cat-S5) (Catapult Sports, Melbourne, Australia) global navigation satellite system standalone receiver with an external antenna and mass of $\sim 67 \mathrm{~g}$. The Cat-S5 has recently been validated [7, 19] with a reported section time error of between 0.1 and $0.2 \mathrm{~s}$ for 20- to 180 -m-long sections, and with errors in section time plateauing for longer sections $[3,19]$. The tracks were 10-15 m wide, located in an area with minimal tree cover and no natural geographical features to interfere with GPS signals. To ensure correct GPS fixing and minimize inaccuracy, the Garmin GPS devices were turned on at least 20 min before the start of testing and blinded for the participants to prevent them using them for any guidance during the time trials.

The participants were timed with an Ipad Air1475 (Apple Inc., California, USA) using the RaceSplitter timing application version 1.7.6 (Makalu Interactive LLC, Delaware, USA). A questionnaire was constructed and used immediately after each of the time trial tests, which, combined with a subsequent interview with each participant directly after the races and visual observations by test leaders, was used to identify where the participants used the G2, G3, G4 skating sub-techniques on the track. Figure 1 is used to help the athletes to point out accurately where the transitions between techniques were made (Fig. 1). All skiers trained almost daily in this track, had previous experience with this procedure and were asked to notice exactly where they switched between different sub-techniques during the simulated competitions.

The track was $5.2 \mathrm{~km}$ (i.e. 2 laps of $2.5 \mathrm{~km}$ plus $200 \mathrm{~m}$ ), which was divided into five 1-km track sections (S1-S5),

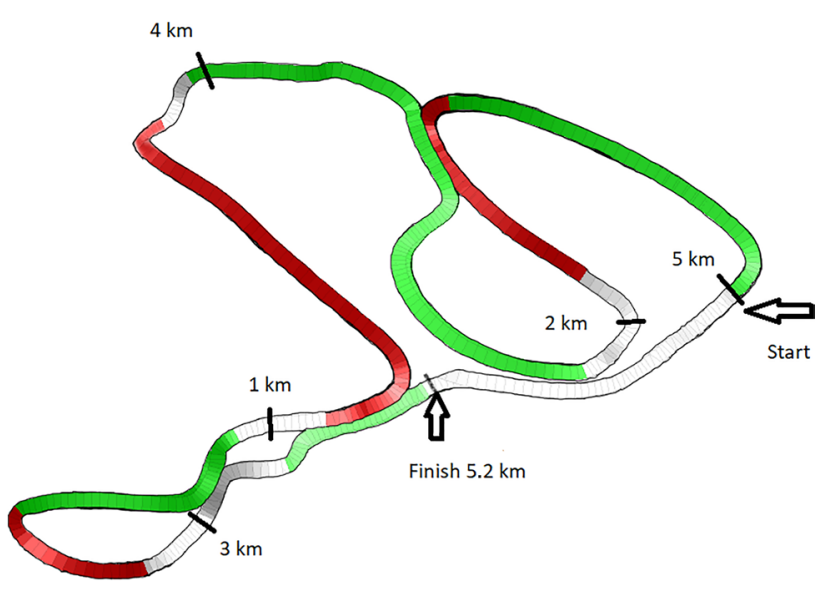

Fig. 1 Profile of the entire 5.2-km track (i.e. 2 laps of $2.5 \mathrm{~km}$ plus $200 \mathrm{~m}$ ) used in the simulated competition. Uphill, flat and downhill terrains are marked with red, white/gray and green colors, respectively 
according to distance from start (see Fig. 1). Section 1 consisted of varied terrain, while section 2 included two hills with mean inclines of $9.3 \%$ and $5.7 \%$ and lengths of $259 \mathrm{~m}$ (26-m climb) and $272 \mathrm{~m}$ (19-m climb), respectively. Section 3 contained varied terrain and included two short hills with 5 and $8 \mathrm{~m}$ climbs in the start of the section and the longest uphill of $396 \mathrm{~m}$ (41-m climb) with a mean incline of $9.3 \%$. Section 4 included the longest downhill section of the track. The two main downhill parts of the track contained inclines of 7.8\% and 5.8\% for lengths of $407 \mathrm{~m}$ and $182 \mathrm{~m}$ in sections 2 and 4, respectively. Section 5 included an uphill of $272 \mathrm{~m}$ (19-m climb where the steepest part was 18.6\%), followed by a $200-\mathrm{m}$ flat segment. The maximal difference in elevation was $41.5 \mathrm{~m}$ with a total climb of $176 \mathrm{~m}$ for the entire track. The time each skier spent in each of the five sections was calculated based on split times. Speed for each section was calculated by dividing the length of a section by the time elapsed within that section.

\section{Statistical Analysis}

The Shapiro-Wilk test confirmed that data did not deviate from normal distribution, and all results are presented as means \pm standard deviation (SD), except for the perceptual responses, which are presented as medians (IQR). To compare the differences in race time, use of sub-technique, as well as physiological and perceptual responses between the two time trials (long poles versus self-selected pole length), a one-way analysis of variance (ANOVA) with repeated measures was performed on each variable. To compare RPE between the two time trials, a Wilcoxon ranked sign test was applied. To identify the differences in time between the time trials for each $\mathrm{km}$, a 2 (condition) $\times 5(1-5 \mathrm{~km})$ ANOVA was performed. Post hoc comparisons with the smallest mean differences were performed for pairwise comparisons between the different sections. The effect size reported in this study was eta squared $\left(\eta^{2}\right)$, where $0.01 \leq \eta^{2}<0.06$ constituted a small effect, $0.06 \leq \eta^{2}<0.14$ a medium effect, and $\eta^{2}>0.14$ a large effect. The level of significance was set at $P<0.05$ for all tests and the analyses were carried out with SPSS Statistics v26 (IBM Corp., Armonk, NY, USA).

\section{Results}

\section{Time Trial Performance}

There were no significant differences in the average time spent on the entire 5-km track between test one in the morning and test two in the afternoon (5.4 s faster), independent of pole length. The total time used for the $5 \mathrm{~km}$ was $7.1 \pm 7.1 \mathrm{~s}$ shorter $\left(F=7.1, P=0.029, \eta^{2}=0.47\right)$ when

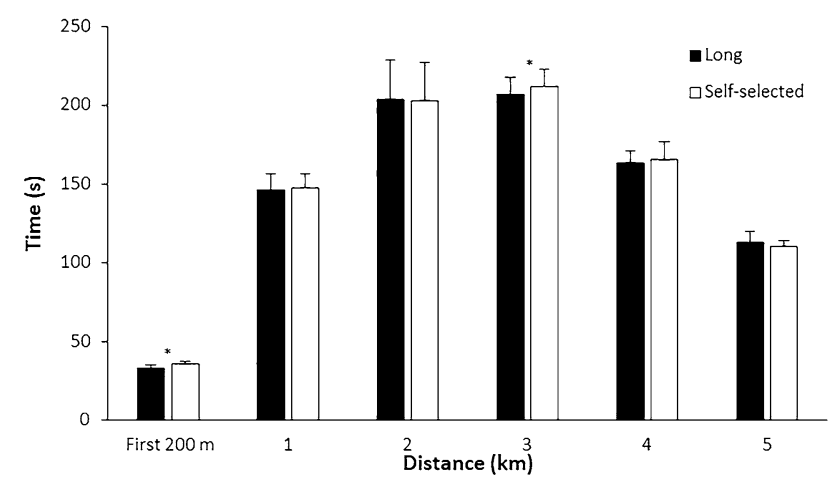

Fig. 2 The influence of pole length on performance in the five sections of the track, as well as the first $200 \mathrm{~m}$. *Indicates a significant time difference between the two pole lengths

Table 1 Physiological and perceptual responses after 5-km crosscountry skiing with self-selected and long $(+7.5 \mathrm{~cm})$ poles

\begin{tabular}{lrr}
\hline & Self-selected & Long poles \\
\hline Peak heart rate (beats/min) & $184.7 \pm 7.4$ & $185.2 \pm 7.7$ \\
Percentage of max heart rate (\%) & $88.2 \pm 3.2$ & $87.9 \pm 3.2$ \\
Average heart rate (beats/min) & $174.0 \pm 7.1$ & $173.4 \pm 7.1$ \\
Percentage of max heart rate (\%) & $93.6 \pm 3.2$ & $93.8 \pm 2.8$ \\
Lactate concentration (mmol/L) & $9.4 \pm 2.0$ & $9.2 \pm 2.3$ \\
RPE (6-20) & $16.8 \pm 1.0 *$ & $15.9 \pm 0.9$ \\
\hline
\end{tabular}

*Indicates a significant difference between the two conditions, $P<0.05$

using the $7.5 \mathrm{~cm}$ longer poles $(867.4 \pm 58.4 \mathrm{~s})$ than selfselected poles $(874.3 \pm 55.8 \mathrm{~s})$. When divided into split times, the third $\mathrm{km}$ that included the longest uphill section of the track was significantly faster (by $\sim 4 \mathrm{~s}$ ) with the longer than with the self-selected poles $(P<0.001)$. In addition, the first $200 \mathrm{~m}$ were also faster (by $\sim 2.7 \mathrm{~s}$ ) with the long poles compared with the self-selected ones $(P<0.05$; Fig. 2$)$. The other sections and the final $200 \mathrm{~m}$ did not differ in performance between the two pole conditions.

No physiological responses differed between the two conditions $\left(F \leq 0.7, P \geq 0.46, \eta^{2} \leq 0.07\right)$. However, a significantly lower RPE $(P=0.046)$ was observed with the long poles compared with the self-selected poles (Table 1). The perception of skiing technique with long poles showed a mean value of $6.2 \pm 1.5$, which was 1.2 points above the score for self-selected poles $(P=0.038)$.

The distribution of different sub-techniques used during the $5 \mathrm{~km}$ was reported differently between the two conditions (Table $2 ; F=28.7, P<0.001, \eta^{2}=0.78$ ). When the skiers were equipped with $7.5 \mathrm{~cm}$ longer poles, they reported using $\mathrm{G} 3$ five percentage points more than the G2 sub-technique; $\mathrm{G} 3$ was used further up on uphill sections before they 
switched to G2. The use of other sub-techniques did not significantly differ.

\section{Discussion}

The main findings in this study were that: (1) long poles enhanced performance compared to self-selected poles, without any alterations in average heart rate, blood lactate concentration and RPE, (2) the use of longer poles led to more use of the G3 sub-technique at the expense of G2 in the uphill sections of the track, and (3) the skiers reported lower RPE and a substantially better perception of the skiing technique when using long poles compared to their selfselected ones.

Our findings of better performance with long compared to self-selected poles in this study are in line with earlier investigations claiming that longer poles enhanced performance and reduced $\mathrm{O}_{2}$ cost during both uphill treadmill roller skiing with the G3 skating technique [29] and uphill double poling while roller skiing on a treadmill $[5,13,14$, 17]. In the present study, the effect of pole length was tested on snow using the skating technique for the first time. We found significant performance improvements with $7.5 \mathrm{~cm}$ longer poles compared to self-selected ones, which were mainly related to enhanced performance in the first $200 \mathrm{~m}$ and during the longest uphill in section 3 . While the superior improvement in the initial $200 \mathrm{~m}$ may be explained by faster acceleration, as previously shown for double poling in the studies by Hansen, Losnegard [8] and Losnegard et al. [14], better section 3 performance was probably due to enhanced performance in the longest uphill section, where skiers reported more use of the G3 technique with longer poles. Since it is assumed that performance in G3 and double poling has clear similarities in the contribution of the upper body to forward propulsion [15], it is reasonable to assume that the improved uphill performance with longer poles in skating on snow found here would be explained by the same mechanisms as previously found in double poling on a treadmill $[5,13,17,30]$. Here, longer poles resulted in lower $\mathrm{O}_{2}$ cost, which was associated with reduced vertical displacement of $\mathrm{CoM}$ and longer poling time.

Table 2 Reported distribution of different techniques (\%) used during the $5-\mathrm{km}$ cross-country race with self-selected and long $(+7.5 \mathrm{~cm})$ poles, exclusive of skating without poles, tucks and turns

\begin{tabular}{llll}
\hline & G3 & G2 & G4 \\
\hline Self-selected & $66.4 \pm 20.5$ & $19.7 \pm 10.3$ & $14.4 \pm 10.4$ \\
Long poles & $71.6 \pm 20.0^{*}$ & $14.9 \pm 9.1^{*}$ & $13.7 \pm 11.0$ \\
\hline
\end{tabular}

*Indicates a significant difference between the two conditions for this sub-technique, $P<0.05$
In a previous publication [29], we reported a higher gross efficiency with long poles compared to self-selected ones in G3 skating at $11 \%$ uphill inclination and $10 \mathrm{~km} / \mathrm{h}$ in roller skiing on a treadmill. This supports the present study, where the skiers reported more use of G3 in uphill sections of the track $(9.3 \%$ incline and average speed of $13.5 \mathrm{~km} / \mathrm{h})$ with longer poles. The effectiveness of using longer poles in uphill skating may be explained by a higher start position for poling and thereby a more upright position with reduced vertical displacement of the CoM, as also found in skating by Torvik et al. [29]. A smaller distance between CoM and pole plant in double poling has also been pointed out by Carlsen et al. [5] and Losnegard et al. [14]. Notably, the enhanced performance found in the present study was found with equal physical strain, and with slightly lower perceived exertion with longer poles.

The possible benefit of long poles in uphill terrain is interesting, since most of the racing time is spent on uphill sections during a race and the greatest time differences between skiers occur there [19]. However, it is not known to what extent lower speed or steeper incline determine the positive effect of increased pole length. Indeed, speed and incline are interrelated, and cycle characteristics and the choice of sub-technique seem to be influenced by both factors when they are isolated. However, the fact that longer poles are most effective at lower speeds, which take place at steeper inclines and at the start when accelerating from zero, indicates that speed might be an important contributor. Because of the lower speeds among women, longer poles might be more beneficial for women than men. While Trøen et al. [30] indicated that this is the case in the classic style, this aspect needs to be further examined in skating.

We expected the last 200-m sprint towards the finishing line to be negatively affected by the long poles due to possible problems with rapid repositioning and maintenance of frequency. However, no such disadvantage was found although none of the skiers was used to skiing with longer poles. In fact, the lack of experience with longer poles indicates that extensive practice with long poles may enhance performance even more. This was exemplified by closer analysis of the data, showing that the only skier who did not benefit from longer poles over the entire $5-\mathrm{km}$ time trial lost $10 \mathrm{~s}$ during the first $2 \mathrm{~km}$. Our communication with the athlete revealed that she struggled to find the right technique for "timing" the pole plants in the first $2 \mathrm{~km}$. However, this participant was also $5 \mathrm{~s}$ faster with the long poles in section 3, which had the longest uphill section of the track. In addition, the two athletes with the lowest FIS points and best performance during the time trial had the greatest effect from the long poles, with 14 and $16 \mathrm{~s}$ improvement. This is not unexpected since these two skiers probably have the best potential for utilizing longer poles, with a well-developed technique and upper-body capacity [25]. However, due to the 
small number of athletes participating in this study, results should be interpreted with caution and further studies with a high number of athletes and a wider range of performance levels would be required to draw firm conclusions.

Despite the lack of practice with longer poles, the participants reported a significantly better perception of skiing technique (i.e., a better feeling) when skiing with longer than with self-selected poles. However, it should be noted that four of the skiers reported that one short 25-m steep uphill section $500 \mathrm{~m}$ from the finishing line was challenging with long poles, due to longer repositioning of the long poles in the G2 sub-technique. Overall, we suggest that with extended practice, longer poles may be a strategy to enhance performance in ski skating. Although Losnegard et al. [14] did not find such an effect of practice with long poles in double poling, it is worth noting that the present study examined skating, where the movement pattern is more complex than in double poling.

\section{Limitations}

Detailed questionnaires and communication with the athletes were used to estimate the use of skating sub-technique in this study. Although there are clear limitations to this method and future approaches should include automatic detection of sub-techniques as implemented previously in the classical style by, e.g., Seeberg et al. [20] and Solli et al. [23], no previous studies had provided valid algorithms for skating $[15,27]$. Although there are clear limitations to this methodology, all the athletes involved in this study had trained for several hundred hours and regularly performed competitions in this track over several years. Accordingly, we believe that they had a sound basis for judging their use of sub-technique, although we do not have reliable data or evidence to state the precise accuracy.

Although there were only nine participants in this study, the data indicated that skiers should consider experimenting with longer poles in ski skating, while a direct translation to competitions may require extensive practice and experience with different types of tracks, snow and weather conditions. In addition, a further understanding of the underlying mechanisms is required, in which information on temporal patterns and joint kinematics in the different skiing sub-techniques with various pole lengths on snow outdoors should be analyzed further.

\section{Conclusions}

This study demonstrates that increasing pole length by $7.5 \mathrm{~cm}$ in on-snow ski skating can be beneficial. Here, the performance improvement induced by longer poles occurred in the initial part of the race and the longest uphill section, which coincided with more use of the G3 sub-technique than G2. Since this took place without any changes in physiological parameters, but with improved perceived feeling and lower RPE with the long poles, we conclude that female XC skiers may enhance performance by choosing longer poles than those preferred in skating today.

Supplementary Information The online version contains supplementary material available at https://doi.org/10.1007/s42978-021-00134-0.

Funding We had no specific funding for this work.

Data Availability All relevant data are within the paper and its supporting information files.

Code Availability Not applicable for the section.

\section{Declarations}

Conflict of interest No conflicts or competing interests exists.

Open Access This article is licensed under a Creative Commons Attribution 4.0 International License, which permits use, sharing, adaptation, distribution and reproduction in any medium or format, as long as you give appropriate credit to the original author(s) and the source, provide a link to the Creative Commons licence, and indicate if changes were made. The images or other third party material in this article are included in the article's Creative Commons licence, unless indicated otherwise in a credit line to the material. If material is not included in the article's Creative Commons licence and your intended use is not permitted by statutory regulation or exceeds the permitted use, you will need to obtain permission directly from the copyright holder. To view a copy of this licence, visit http://creativecommons.org/licenses/by/4.0/.

\section{References}

1. Bengtsson BE. Cross-country skating: how it started. 2018. https://www.skiinghistory.org . Accessed 2 Mar 2018.

2. Bilodeau B, Roy B, Boulay MR. A comparison of three skating techniques and the diagonal stride on heart rate responses and speed in cross-country skiing. Int J Sports Med. 1991;12(1):71-6.

3. Bolger CM, Kocbach J, Hegge AM, Sandbakk Ø. Speed and heartrate profiles in skating and classical cross-country skiing competitions. Int J Sports Physiol Perform. 2015;10(7):873.

4. Boulay MR, Rundell KW, King DL. Effect of slope variation and skating technique on velocity in cross-country skiing. Med Sci Sports Exerc. 1994;27(2):281-7.

5. Carlsen CH, Rud B, Myklebust H, Losnegard T. Pole lengths influence $\mathrm{O} 2$-cost during double poling in highly trained crosscountry skiers. Eur J Appl Physiol. 2018;118(2):271-81.

6. FIS. The International Ski Competition Rules (ICR: Book II Cross- Country) 2017. https://www.fis-ski.com/en/inside-fis/ document-library/cross-country-documents. Accessed 7 May 2017.

7. Gløersen $\varnothing$, Kocbach J, Gilgien M. Tracking performance in endurance racing sports: evaluation of the accuracy offered by three commercial GNSS receivers aimed at the sports market. Front Physiol. 2018;9:1425. 
8. Hansen E, Losnegard T. Pole length affects cross-country skiers' performance in an 80-m double poling trial performed on snow from standing start. Sports Eng. 2010;12(4):171-8.

9. Holmberg H-C, Lindinger S, Stöggl T, Eitzlmair E, Müller E. Biomechanical analysis of double poling in elite cross-country skiers. Med Sci Sports Exerc. 2005;37:807-18.

10. Ingjer F. Maximal oxygen uptake as a predictor of performance ability in women and men elite cross-country skiers. Scand J Med Sci Sports. 1991;1:25-30.

11. Kvamme B, Jakobsen V, Hetland S, Smith G. Ski skating technique and physiological responses across slopes and speeds. Eur J Appl Physiol. 2005;95(2-3):205-12.

12. Lindinger S, Stoeggl T, Mueller E. Biomechanical charateristics of further developed classical and skating techniques in cross-country skiing sprint competitions. J Biomech. 2006;39:S187-S187.

13. Losnegard T, Myklebust H, Skattebo $\varnothing$, Stadheim HK, Sandbakk $\varnothing$, Hallén J. The influence of pole length on performance, O2 cost, and kinematics in double poling. Int J Sports Physiol Perform. 2017;12(2):211-7.

14. Losnegard T, Tosterud OK, Trøen E, Carlsen CH, Paulsen G, Rud B. The influence of pole lengths on $\mathrm{O} 2$-cost, kinematics, and performance in double poling at high speeds before and after a training period with long poles. Eur J Appl Physiol. 2019;119(11-12):2579.

15. Myklebust H, Losnegard T, Hallen J. Differences in V1 and V2 ski skating techniques described by accelerometers. Scand J Med Sci Sports. 2014;24(6):882-93.

16. Nilsson J, Jakobsen V, Tveit P, Eikrehagen O. Pole length and ground reaction forces during maximal double poling in skiing. Sport Biomech. 2003;2(2):227-36.

17. Onasch F, Killick A, Herzog W. Is there an optimal pole length for double poling in cross country skiing? J Appl Biomech. 2017;33(3):197-202.

18. Sandbakk $\emptyset$, Holmberg H-C. Physiological capacity and training routines of elite cross-country skiers: approaching the upper limits of human endurance. Int J Sports Physiol Perform. 2017;12(8):1003-11.

19. Sandbakk Ø, Losnegard T, Skattebo Ø, Hegge AM, Tønnessen E, Kocbach J. Analysis of classical time-trial performance and technique-specific physiological determinants in elite female cross-country skiers. Front Physiol. 2016;7:326.

20. Seeberg T, Tjønnås J, Rindal O, Haugnes P, Dalgard S, Sandbakk $\varnothing$. A multi-sensor system for automatic analysis of classical crosscountry skiing techniques. Sports Eng. 2017;20(4):313-27.
21. Skattebo Ø, Losnegard T, Stadheim HK. Double poling physiology and kinematics of elite cross-country skiers: specialized longdistance versus all-round skiers. Int J Sports Physiol Perform. 2019;14:1199.

22. Smith G. Cross-country skiing: technique, equipment and environmental factors affecting performance. In: Zatsiorsky V, editor. Biomechanics in sport. IOC encyclopedia of sports medicine series. Oxford: Blackwell Science; 2000. pp. 247-70.

23. Solli GS, Kocbach J, Seeberg TM, Tjønnås J, Rindal OM, Haugnes P, Torvik P- $\varnothing$, Sandbakk $\emptyset$. Sex-based differences in speed, sub-technique selection, and kinematic patterns during low- and high-intensity training for classical cross-country skiing. PLoS ONE. 2018;13(11):e0207195.

24. Stöggl LT, Holmberg H-C. Force interaction and 3D pole movement in double poling. Scand J Med Sci Sports. 2011;21:e393-404.

25. Stöggl LT, Holmberg LH-C. Double-poling biomechanics of elite cross-country skiers: flat versus uphill terrain. Med Sci Sports Exerc. 2016;48(8):1580-9.

26. Stöggl T, Müller E, Lindinger S. Biomechanical comparison of the double-push technique and the conventional skate skiing technique in cross-country sprint skiing. J Sports Sci. 2008;26(11):1225-33.

27. Stöggl T, Holst A, Jonasson A, Andersson E, Wunsch T, Norström C, Holmberg HC. Automatic classification of the sub-techniques (gears) used in cross-country ski skating employing a mobile phone. Sensors. 2014;14(11):20589-601.

28. Tønnessen E, Haugen TA, Hem E, Leirstein S, Seiler S. Maximal aerobic capacity in the winter Olympic endurance disciplines: Olympic medal benchmarks for the time period 1990-2013. Int J Sports Physiol Perform. 2015;10(7):835-9.

29. Torvik P- $\varnothing$, Von Heimburg E, Sende T, Welde B. The effect of pole length on physiological and perceptual responses during G3 roller ski skating on uphill terrain. PLoS One. 2019;14:e0211550.

30. Trøen E, Rud B, Karlsson Ø, Carlsen CH, Gilgien M, Paulsen G, Tosterud OK, Losnegard T. Pole length's influence on performance during classic-style snow skiing in well-trained crosscountry skiers. Int J Sports Physiol Perform. 2020;15(6):884.

31. Vandbakk K, Welde B, Kruken AH, Baumgart J, Ettema G, Karlsen T, Sandbakk $\emptyset$. Effects of upper-body sprint-interval training on strength and endurance capacities in female crosscountry skiers. PLoS One. 2017;12(2):e0172706. 